\title{
Adnexal Diseases
}

\author{
Andrea Rockall and Rosemarie Forstner
}

\section{Learning Objectives}

- To know the most common benign ovarian lesions

- To be aware of the indications for MRI of adnexal masses

- To know the sequences for basic and fully optimized MRI of adnexal masses

- To be able to use an algorithmic approach to characterize adnexal masses

\subsection{Introduction}

Adnexal masses are common and may present symptomatically with pelvic or abdominal pain or may be identified incidentally during the course of imaging for another indication. The majority of adnexal masses are benign, but a small number will be borderline tumors or invasive cancer. In the case of a benign lesion, the aim is to reassure the patient and manage according to the clinical need, without subjecting the patient to over-extensive or inappropriate surgery [1]. Conversely, in cases that demonstrate features with a higher likelihood of ovarian malignancy, referral to a specialist center will ensure the best possible outcome for the patient.

One major difficulty is that undertaking a biopsy to confirm the nature of an adnexal mass is not advised, if there is no peritoneal disease beyond the ovary. Biopsy could result in spillage of a potentially confined tumor and increase stage and risk for patient. Therefore, the clinical decision on how

\footnotetext{
A. Rockall

Consultant Radiologist, Department of Radiology, The Royal Marsden NHS Foundation Trust, London, UK

Professor of Radiology, Faculty of Medicine, Imperial College London, UK

R. Forstner $(\bowtie)$

Faculty of Medicine, Imperial College London, London, UK e-mail: R.Forstner@salk.at
}

aggressively to manage the ovarian mass relies on the available clinical and image findings, rather than histology.

The clinical context at the time of presentation is relevant. The age and menopausal status of the patient and the presence of acute or chronic pain or fever may inform the interpretation of image findings. In premenopausal women with pain, a pregnancy test is indicated in order to rule out possible ectopic pregnancy as a cause of an adnexal mass.

Ultrasound is the first-line imaging modality, allowing the characterization of most adnexal masses. MRI is indicated in the characterization of sonographically indeterminate masses, whereas CT is indicated for further staging of a suspected ovarian cancer on US.

\subsection{Imaging Modalities to Assess an Adnexal Mass}

\subsubsection{Ultrasound (US)}

Optimally, transvaginal ultrasound (TVUS) and transabdominal ultrasound (TAUS) are combined to provide characterization of the adnexal mass and its differentiation from the uterus or other pelvic abnormalities. TAUS also provides information of ancillary findings that may be crucial for the diagnosis, e.g., presence of peritoneal implants, ascites, pleural effusion, and lymph node enlargement, and assessment of the kidneys and the bowels. For characterization of an adnexal mass, the following imaging features indicative of malignancy have been widely used: wall irregularity, thick septations $(3 \mathrm{~mm})$, papillary projections, solid components, and large size $(4 \mathrm{~cm})$ [1-3]. Unfortunately these features overlap with those of benign pathologies, e.g., tubo-ovarian abscess, corpus luteum cyst, endometriomas, and some rare benign tumors. Recently, the IOTA simple rules model has been introduced to preoperatively assess adnexal masses with US. Five sonographic imaging features each defining the B (benign) and M (malignant) categories are used to distinguish 
between malignant and benign adnexal masses [4-6]. Thus, in expert hands approximately $78-80 \%$ of complex adnexal masses can be diagnosed with US alone. Limitations include mostly benign solid masses, e.g., endometriomas, fibromas, thecomas, and rare other ovarian tumors.

\subsubsection{Magnetic Resonance Imaging (MRI)}

MRI is recommended as a second-line imaging technique to evaluate adnexal masses that remain indeterminate on US [7]. MRI combines excellent contrast resolution and lack of nonionizing radiation, which is of utmost importance in children and in the reproductive age. It is most useful in women with a low pretest probability of malignancy of the adnexal mass. Studies show high diagnostic sensitivity (67$100 \%)$ and specificity (77-100\%) [8-16].

Typical MRI protocols include T2-weighted imaging in two planes, usually the sagittal $\mathrm{T} 2$-weighted imaging providing overview of the uterus and the ovaries and a transaxial T1- and high-resolution fast spin echo (FSE) T2-weighted imaging sequence covering in thin slices the ovaries [7]. If the ovaries are not seen, oblique axial T2-weighted imaging taken along the long axis of the uterus will visualize the ovaries in atypical position along their ovarian axis. In equivocal cases, this plane or alternatively a coronal plane also serves in solving important differential diagnostic issues, e.g., the nonovarian origin of a parauterine mass (i.e., pedunculated uterine leiomyoma). Features of subserous uterine leiomyomas present presence of bridging vessels between the mass and the myometrium or the claw sign of the uterine myometrium. In contrast, demonstrations of follicles within or in the periphery of the mass and the ovarian beak sign are typical features of ovarian origin of a pelvic mass [7].

The majority of adnexal lesions can be characterized by their specific signal characteristics on T1- and T2-weighted imaging. Simple fluid has homogeneous low signal on T1-weighted imaging and high SI on T2-weighted imaging. Fat and hemorrhage have high SI on T1-weighted imaging. Fat suppression (FS) on T1-weighted imaging is used to differentiate fat-containing dermoids from hemorrhagic cystic lesions [7]. In solid and in cystic adnexal masses, characterization is best performed by complementing the basic sequences with DWI and intravenous administration of gadolinium $[11,15,16]$. For characterization of adnexal masses, the recommended high $b$ value is between 800 and $1200 \mathrm{~mm} / \mathrm{s}^{2}$. Optimally, dynamic multiphase contrast-enhanced (DCE) MRI is used to characterize adnexal masses, as the time-intensity curve of a solid aspect of the mass in relation to the myometrium will provide important differential diagnostic information. Subtraction images are essential to evaluate enhancing aspects within hemorrhagic lesions, e.g., of nodules within endometriomas. Fasting for $4 \mathrm{~h}$, application of antiperistaltic medication, and examination with a medium full bladder are used to optimize the image quality [7].

\subsubsection{Computed Tomography (CT)}

In clinical practice contrast-enhanced $\mathrm{CT}$ is widely used to assess abdominal pathologies, and usually this is accomplished by covering the abdomen and the pelvis. For characterization of a sonographically indeterminate mass, CT is not recommended, as it is inferior to both MRI and US to assess solid and hemorrhagic masses. CT is, however, the modality of choice to stage suspected ovarian cancer [17]. Another role of $\mathrm{CT}$ is in the emergency setting, where it is widely used to assess the acute abdomen, for both gynecological and non-gynecological causes [18].

\subsubsection{Positron Emission Tomography/ Computed Tomography (PET/CT)}

PET/CT using fluoro-2-deoxy-D-glucose (FDG) has currently no role in characterizing of adnexal lesions. Physiological uptake in premenopausal age in normal ovaries and in corpus luteum cysts is a well-known cause of misinterpretation [19]. FDG uptake can also be seen in benign ovarian tumors, such as dermoids, as well as in inflammatory and infectious processes. However, in postmenopausal age FDG avid uptake in the ovaries is typically associated with malignant disease. Currently the role of PET/CT includes assessment of suspected recurrence in two scenarios in patients with ovarian cancer: a) in biochemically suspected recurrence and no evidence of disease with other imaging techniques or b) to exclude systemic disease in surgical potential candidates with localized disease.

\subsection{Adnexal Masses on MRI}

Imaging characteristics of benign and malignant ovarian masses are summarized in Table 8.1.

\subsubsection{Benign Cystic Ovarian Masses}

\subsubsection{Ovarian Cysts}

In reproductive age physiological ovarian cysts constitute the vast majority of adnexal masses. Ovarian follicles and follicular cysts share the same imaging features of thin-walled lesions with watery contents, but per definition the term cyst should be reserved only to those larger than $3 \mathrm{~cm}$ in size [1]. Due to their high prevalence in premenopausal age, cysts ranging from 3 to $5 \mathrm{~cm}$ do not require further follow-up, unless they are symptomatic [1]. Ovarian cysts include either follicular cysts or corpus luteum cysts. The latter derive from failure of regression or hemorrhage into the corpus luteum. Corpus luteum and corpus luteum cysts typically have a wellvascularized thicker wall that may be crenulated and often contain blood products. Thus they display a lacelike appear- 


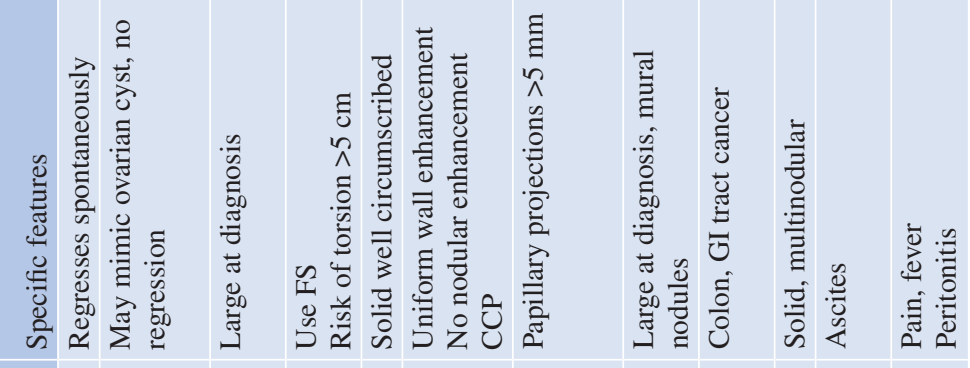

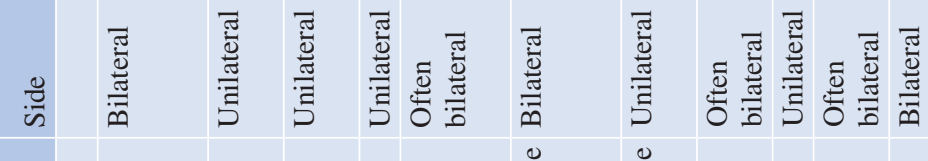

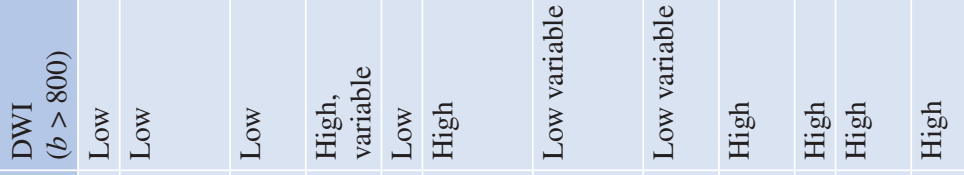

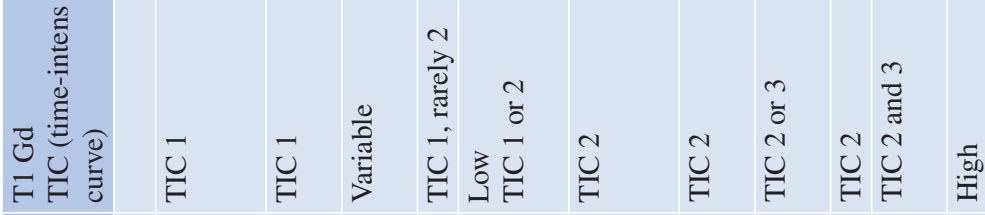

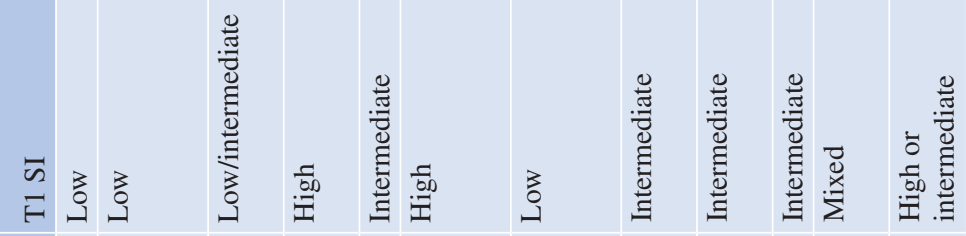

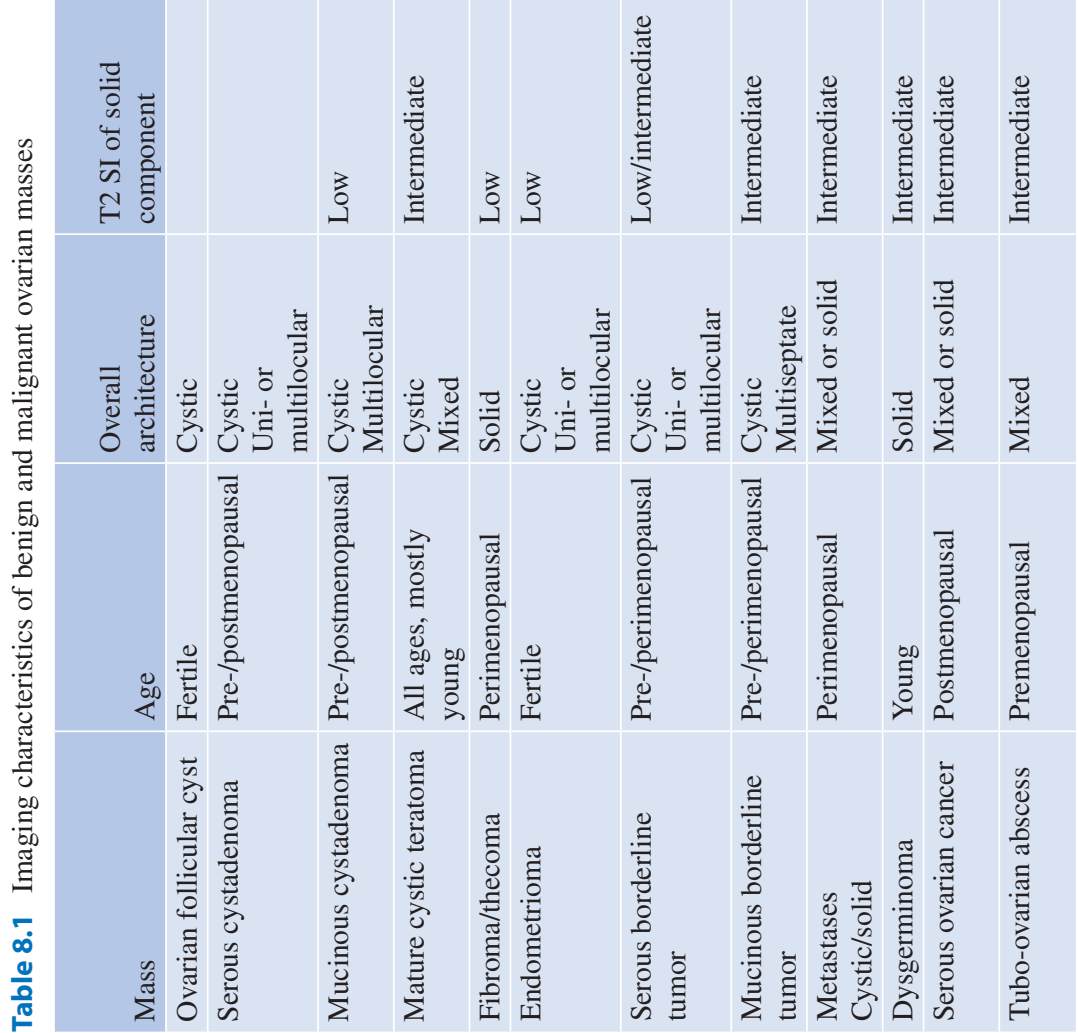


ance in US and not watery contents but signal intensity of blood products on MRI (high SI on T1-weighted imaging and FS T1-weighted imaging or hemosiderin). Typical is unilaterality and regression in a 2-3 months interval.

\subsubsection{Endometriomas}

Endometriomas present endometriotic cysts of the ovaries and constitute the most common manifestation of endometriosis. Most commonly they present bilateral thick-walled unior multiloculated cystic lesions containing blood products. Pathognomonic features include high SI on T1-weighted imaging and FS T1-weighted imaging and low SI on T2-weighted imaging (shading) (Fig. 8.1) [20]. The T2 dark spot sign presenting focal mural clot is another typical feature [21]. As in unilocular hemorrhagic cysts the distinction between endometriomas and hemorrhagic cysts may sometimes be difficult, follow-up US is recommended. Only functional cysts will resolve. Bilaterality and multicystic appearance favor endometrioma. Adhesions with low SI bands and distortion of the ovaries, the uterus, or the bowel are other typical findings in endometriosis. As deep endometriosis, particularly involving the posterior fornix or the rectosigmoid colon, is often associated with endometriomas, this area should also be scrutinized.

\subsubsection{Benign Teratomas (Mature Cystic Teratomas or Dermoid Cysts)}

The typical finding of a mature teratoma is that of a unilocular cystic lesion containing fatty elements. Often a mural nodule, the Rokitansky nodule or dermoid plug, projects into the lesion. It is composed of a variety of tissues and typically demonstrates fat and calcifications, representing teeth or bones. A minority (approx. 15\%) of mature cystic teratomas will demonstrate no fat or only small foci of fat within the wall or the Rokitansky nodule [22].

As US features of dermoids may overlap with those of other entities, such as endometriomas or ovarian carcinomas, CT (Fig. 8.2) or MRI (Fig. 8.3) is performed in suspected dermoids, as they allow a specific diagnosis. In both
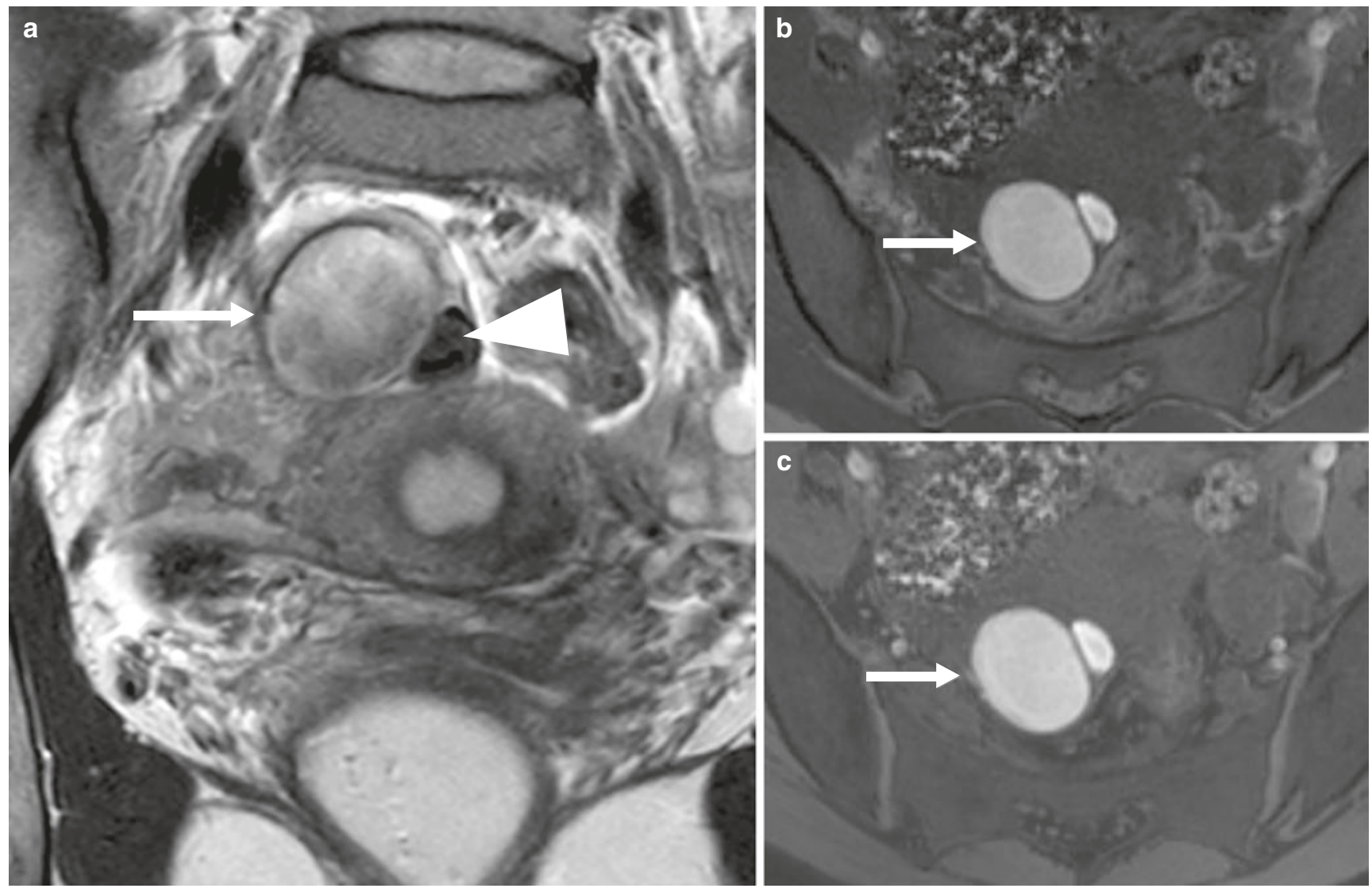

Fig. 8.1 Right ovarian endometrioma. Coronal FSE T2-weighted imaging shows a septate right cystic ovarian lesion with thin walls (a) (arrow). It demonstrates diffuse moderate hypointensity and adjacent very low SI (shading effect) relating to chronic hemorrhage. (b)
Transaxial T1-weighted imaging and (c) FS T1-weighted imaging (arrow) demonstrate high SI characteristic of internal blood products. There is no evidence of internal or mural nodularity 
of these techniques, identification of fat is the pivotal finding. On MRI, the fatty component will demonstrate high SI on both T1- and T2-weighted imaging, with the lipid-laden cyst fluid demonstrating a similar high SI on T1-weighted imaging and intermediate SI on T2-weighted imaging. Chemical shift artifact is often seen at the fat-fluid interface in dermoid cysts as bright or dark bands along the frequency-encoding gradient on T2-weighted imaging. Use of FS T1-weighted imaging enables dermoid cysts (which drop the SI) to be distinguished from hemorrhagic lesions or endometriomas (which retain high SI). In lipid poor dermoids chemical shift imaging techniques may assist in defining the correct diagnosis. Due to their very slow growth, surveillance is a treatment option in dermoids smaller than $5 \mathrm{~cm}$ in size.

Rarely, malignant transformation may develop within a dermoid cyst; most frequently this is squamous cell cancer in a woman of advanced age. Capsular penetration is the most reliable sign to establish this extremely rare complication [23].

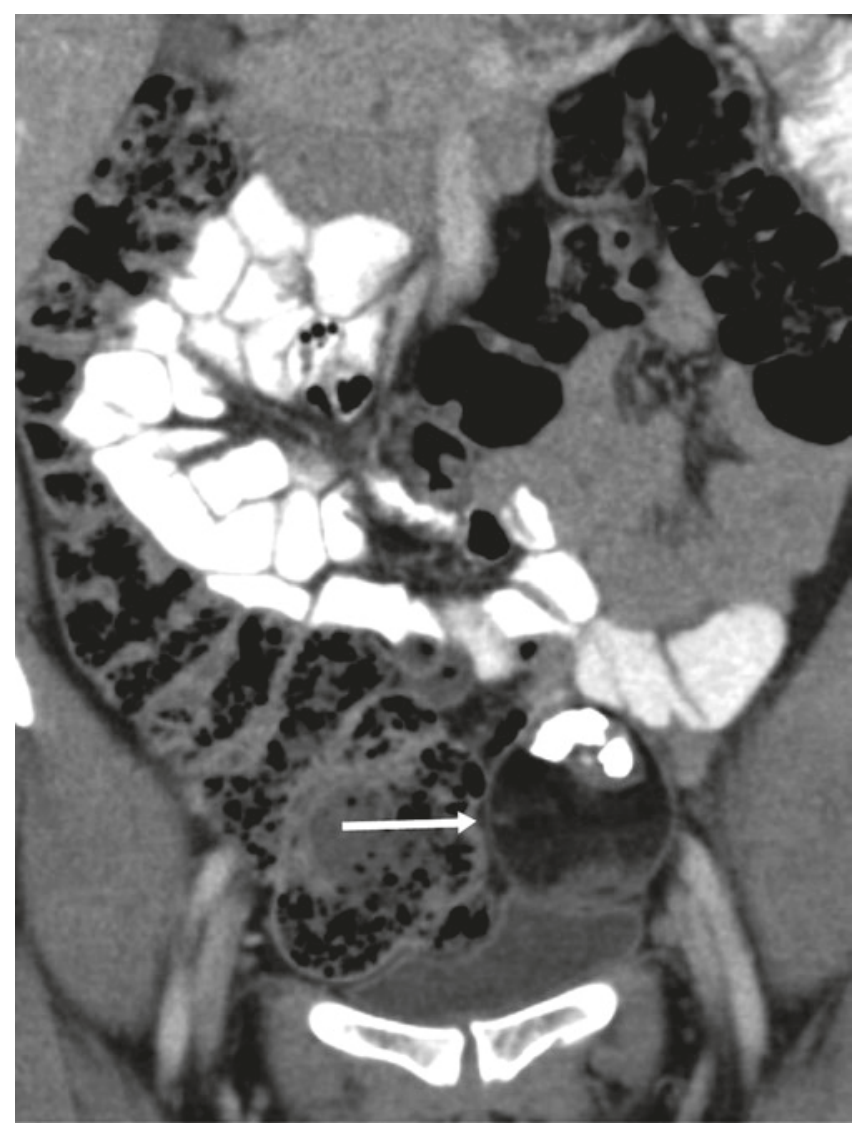

Fig. 8.2 Benign teratoma. CT shows a well-defined cystic lesion with thin walls (arrow). Its fatty contents allow the specific diagnosis of a benign teratoma. Calcification presenting teeth within its superior aspect present another other common finding

\subsubsection{Cystadenomas}

Cystadenomas present common epithelial ovarian tumors, accounting for up to $50 \%$ of tumors in reproductive age and for up to $80 \%$ of benign ovarian tumors in postmenopausal age [24]. Both have now been recognized as precursors of ovarian cancer and may slowly transform to borderline tumors and to invasive ovarian cancer [25]. Serous cystadenomas are more common than mucinous cystadenomas. Although an overlap exists, imaging features aid in the differentiation of serous from mucinous cystadenomas [24]. In general, both entities are thin-walled cystic lesions (Fig. 8.4). Serous cystadenomas follow the pattern of a simple cyst or of a multiseptate mass, whereas a mucinous cystadenoma is typically a large multilocular lesion with locules of various fluid contents [26]. On MRI, cysts in mucinous tumors demonstrate various signal intensities on T1- and T2-weighted imaging, giving rise to the so-called "stained-glass" appearance. This is because on T1-weighted imaging locules with watery mucin generate lower SI than locules with thicker mucin. The opposite is generated on T2-weighted imaging. Cystadenomas can be differentiated from hydrosalpinx due to their complete septations on multiplanar imaging.

\subsubsection{Benign Solid Ovarian Tumors}

These present a rare subgroup of ovarian tumors mostly comprising fibromas, thecomas, and Brenner tumors. They are usually unilateral solid ovarian tumors found in perimenopausal age [27]. In clinical practice they have to be differentiated from their mimickers, the more common pedunculated uterine leiomyomas. Signs defining the ovarian origin are pivotal for the differentiation. Due to their composition of fibrous tissue, they usually display typical features of benign adnexal masses with solid elements displaying very low SI on T2-weighted imaging. In degenerative changes including edema, cystic changes, or torsion, their differentiation from malignant masses is challenging. However, when such a solid lesion demonstrates low SI on DWI using a high $b$ value, malignancy can be ruled out $[12,16]$. Mild and slow gadolinium (type 1) enhancement pattern is seen in the majority of cases. The triad of an ovarian fibroma, ascites, and pleural effusion constitutes the benign Meigs syndrome, which can be associated with elevated CA-125. Unlike fibromas, more than half of thecomas express estrogenes and may present with uterine bleeding, endometrial hyperplasia, or endometrial cancer [27]. Dense amorphous calcifications are a typically feature in Brenner tumors in CT. 

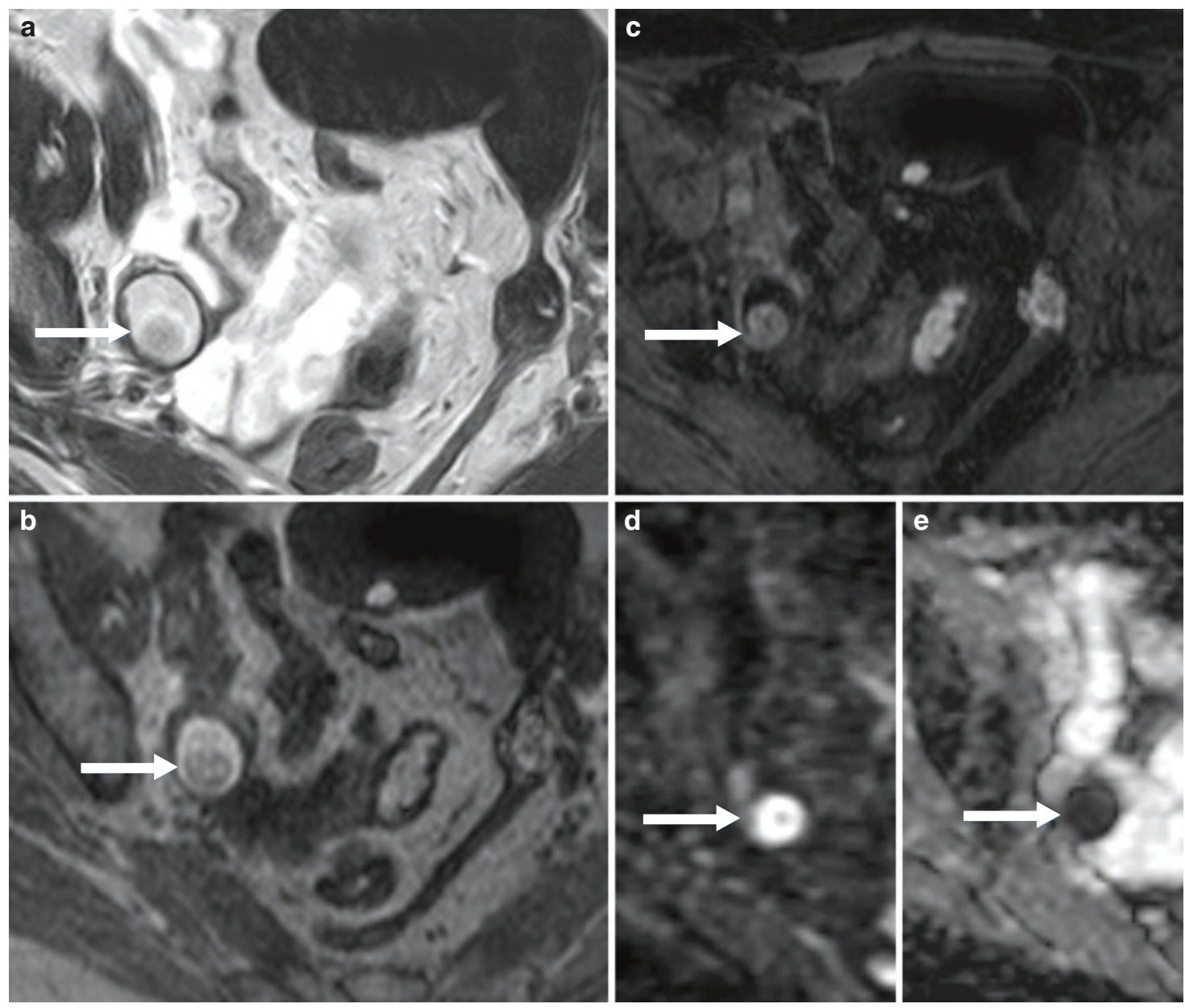

Fig. 8.3 Benign teratoma. MRI shows a right adnexal mass of $2 \mathrm{~cm}$ in size (arrow). It displays high SI on transaxial FSE T2-weighted imaging (a) and internal chemical shift artifact. High SI on T1-weighted imag-

istic of fatty contents. A nodule, the Rokitansky nodule, is seen at its posterior aspect in a-c. On DWI using b1000 s/mm (d) and on ADC (e), the lesion displays diffusion restriction that is due to its sebaceous ing (b) and loss of signal on FS T1-weighted imaging (c) are charactercontents and should not be misdiagnosed as sign of malignancy

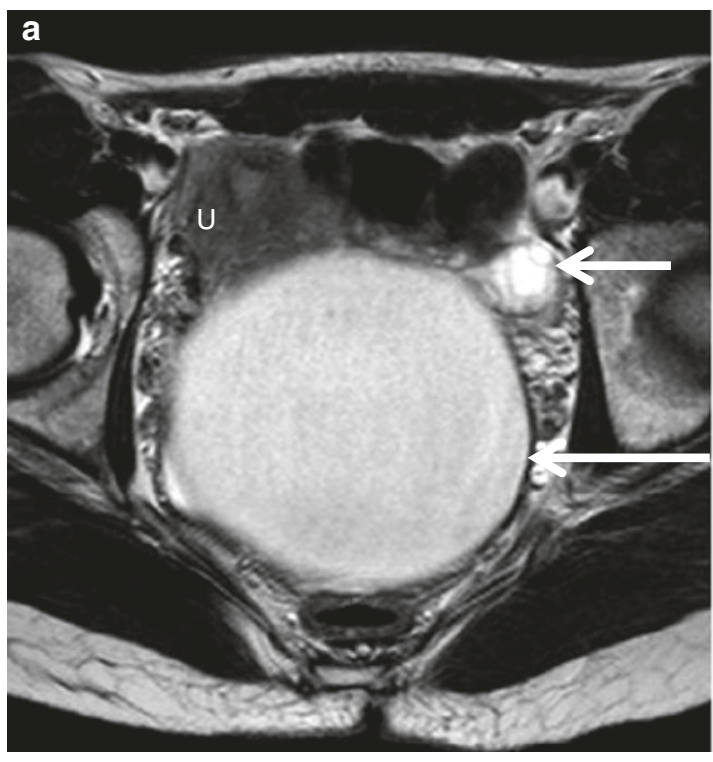

Fig. 8.4 Benign ovarian cystadenoma. Axial T2 (a) and Axial T1 fat sat with contrast (b). There is a normal left ovary with follicles (short arrow). There is a unilocular thin-walled cyst with no solid tissue fol-

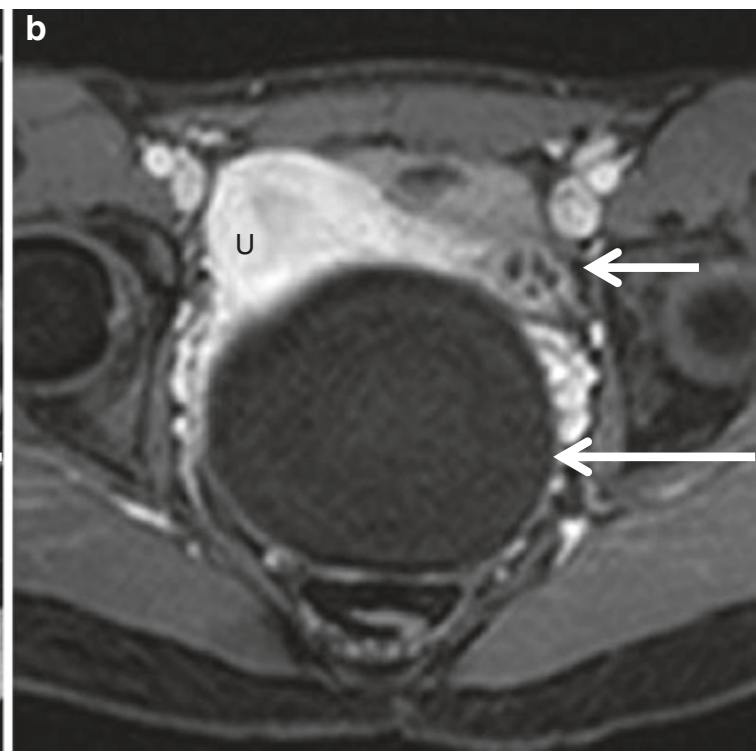

lowing contrast administration (long arrow). The uterus (U) enhances brightly. AdnexMR score is 2 


\subsubsection{Borderline and Malignant Ovarian Tumors}

Borderline tumors account for approximately $15 \%$ of ovarian tumors. The vast majority of ovarian malignant lesions include invasive epithelial ovarian cancer and metastases. The latter constitute $15 \%$ of malignant ovarian masses and originate mostly from breast and the GI tract cancer. In contrast, sex cord-stromal tumors (granulosa cell and SertoliLeydig cell tumors) and malignant germ cell tumors are rare tumors, mostly found in young age.

Borderline tumors are now considered as precursor lesions that gradually progress to different subtypes of epithelial ovarian cancer type II. Serous high-grade ovarian cancer, the most common ovarian cancer, shares features of tubal and primary peritoneal cancer. It seems to develop de novo rapidly and is characterized by rapid dissemination into the peritoneal cavity and usually presents with tumor dissemination outside the pelvis and ascites at diagnosis [28].

Likelihood of malignancy increases with the presence of septations, mural nodules, and papillary projections (Fig. 8.5). MRI features suspicious for malignancy include mass $4 \mathrm{~cm}$, cystic lesion with solid component, irregular wall thickness of $3 \mathrm{~mm}$ or more, septal thickness of $3 \mathrm{~mm}$ or more, presence of papillary projections or nodules, and presence of solid mass with necrosis and early, bright contrast enhancement [3, $16,29]$. In addition, ancillary criteria for suspected malignancy include direct local tissue invasion, peritoneal deposits, enlarged lymph nodes, and ascites. Dynamic CE (DCE)
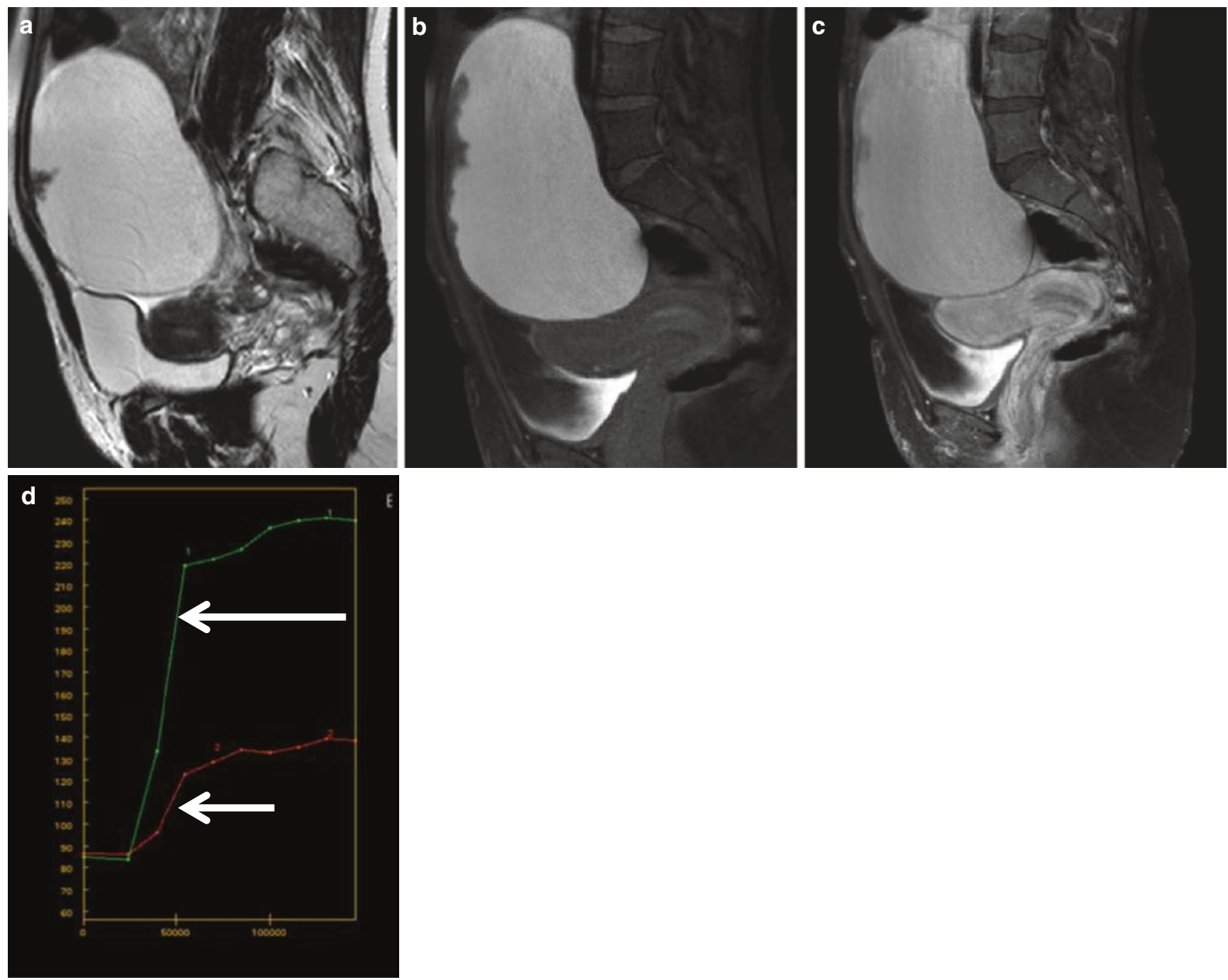

Fig. 8.5 Serous borderline ovarian tumor. (a) T2-weighted image demonstrates a papillary formation arising from the anterior wall of a unilocular cyst. (b, c) T1 fat-saturated images before and after contrast demonstrate enhancement of multiple papillary structures at a different position in the cyst. (d) The time-intensity curve in the solid tissue is type 2 (short arrow is the enhancement curve of the papillary formation, and long arrow is the enhancement curve of the outer myometrium). AdnexMR score is 4 


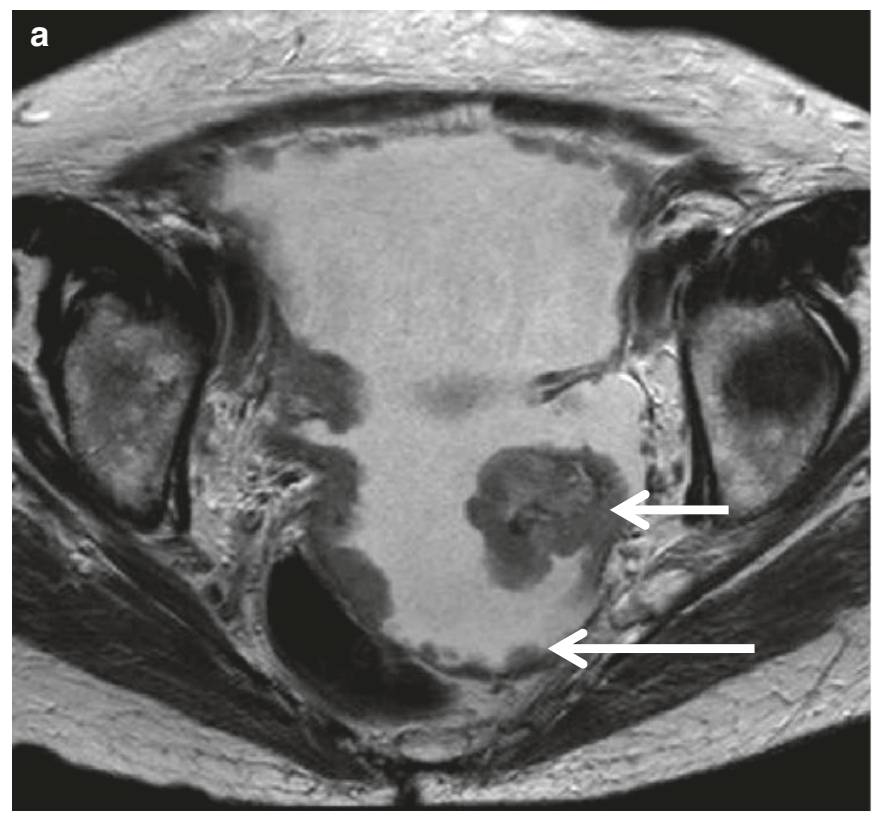

Fig. 8.6 Disseminated peritoneal disease in tubo-ovarian cancer on MRI (a) axial T2 and (b) axial b1000 diffusion image. Left ovarian mass (short arrow) and peritoneal disease (long arrow). The solid tissue

MRI is used for risk stratification in cystic and solid adnexal masses. Time-intensity curves types 1,2, and 3 obtained from the solid aspects of such masses are associated with benign, borderline, and invasive tumors [16]. Gadolinium also improves peritoneal and omental implant detection in case of ovarian carcinoma. Due to the overlap of ADC values, DWI is less useful to characterize indeterminate adnexal masses, but it is pivotal to assess peritoneal carcinomatosis [14-17].

$\mathrm{CE}-\mathrm{CT}$ remains the technique of choice for staging ovarian carcinoma [17] (Figs. 8.6 and 8.7).

\subsection{Risk Stratification of Adnexal Masses Using the AdnexMR Score}

When interpreting an adnexal mass on MRI, an algorithmic approach is strongly advocated. A risk stratification can also be applied (Table 8.2) [16].

1. The first step is to identify if indeed there is a pelvic mass, as occasionally a physiological ovarian mass may have resolved by the time of MRI. If there is a pelvic mass, then it is important to be sure whether it is arising from the ovary. Non-ovarian masses, such as leiomyomata, peritoneal inclusion cysts, or gastrointestinal stromal tumors, or some extraperitoneal lesions, such as Schwannoma, may be misinterpreted as adnexal on US. It is most important to check for the presence of normal ovaries, and this can also be challenging on MRI when a large mass fills the pelvis. It may be helpful to follow the

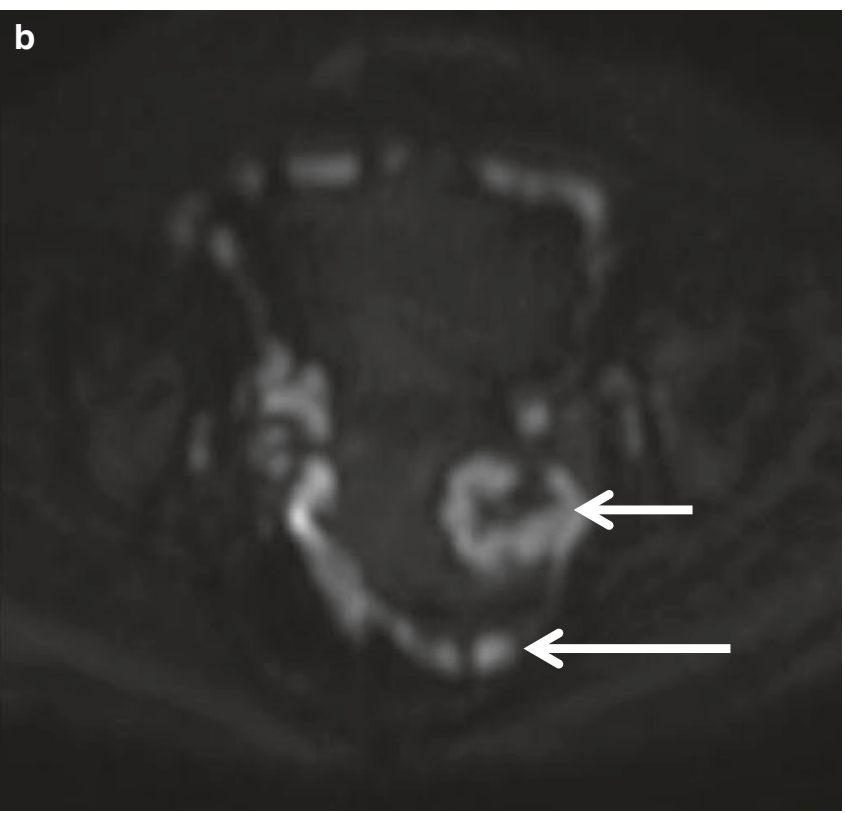

of the ovarian mass is intermediate in signal intensity. The ovarian solid tissue and the peritoneal disease are very bright on the diffusion image. AdnexMR score is 5 due to the presence of peritoneal disease

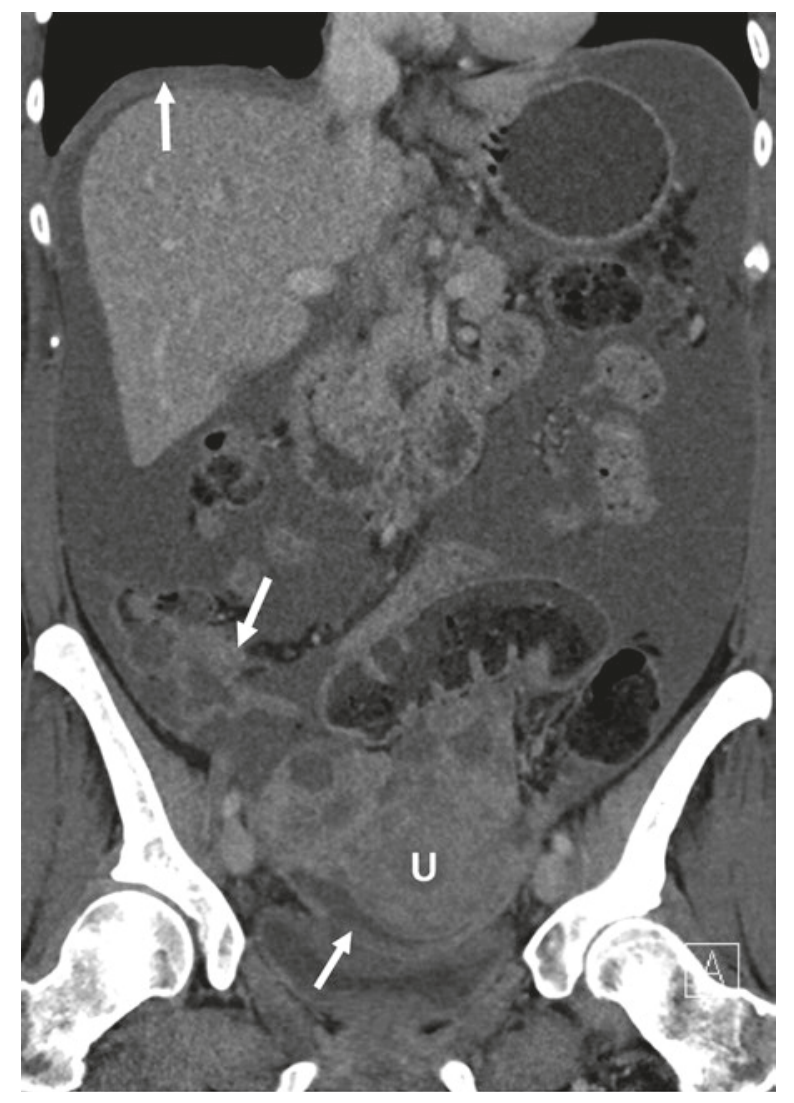

Fig. 8.7 Ovarian cancer. CT demonstrates a solid and cystic ovarian mass adjacent to the uterus $(u)$. Findings indicative of peritoneal dissemination in the pelvis and abdomen include large amounts of ascites and peritoneal deposits at the right diaphragm, bowel surface, and pelvic peritoneum (arrows) 
Table 8.2 AdnexMR scoring system [16]

\begin{tabular}{|l|l|}
\hline AdnexMR score & Criteria \\
\hline $\begin{array}{l}\text { 1. No ovarian mass } \\
\text { mass }\end{array}$ & No mass \\
\hline $\begin{array}{c}\text { 3. Probably benign } \\
\text { mass }\end{array}$ & $\begin{array}{l}\text { Purely cystic mass } \\
\text { Purely endometriotic mass } \\
\text { Purely fatty mass } \\
\text { Absence of wall enhancement } \\
\text { Low } b=1000 \text { sec.mm }{ }^{2} \text { and low T2-weighted } \\
\text { signal intensity within solid tissue }\end{array}$ \\
\hline $\begin{array}{c}\text { 4. Indeterminate MR } \\
\text { mass }\end{array}$ & $\begin{array}{l}\text { Aurve type } 1 \text { within solid tissue } \\
\text { Curve type 2 within solid tissue }\end{array}$ \\
\hline $\begin{array}{c}\text { 5. Probably } \\
\text { malignant mass }\end{array}$ & $\begin{array}{l}\text { Peritoneal disease } \\
\text { Curve type 3 within solid tissue }\end{array}$ \\
\hline
\end{tabular}

gonadal vessels down into the pelvis, and the presence of follicles or a corpus luteum cyst, if present, is confirmatory. If two normal ovaries are identified, then the AdnexMR score is 1, and the MRI should focus on characterizing the non-ovarian mass.

2. If there is an ovarian lesion, then the next question is whether the cyst has benign features. This could include a unilocular cyst with no enhancing solid tissue, a typical endometrioma, or a mature cystic teratoma. Lesions with no wall enhancement or lesions with solid tissue that are homogeneously low signal intensity on both $\mathrm{T} 2$ and high $\mathrm{b}$ value DWI, with low enhancement, are almost certainly benign. These lesions all fall into an AdnexMR score of 2.

3. If an ovarian lesion does not comply with the above findings, then the presence and enhancement pattern of solid tissue is important. Solid tissue is defined as enhancing tissue in an irregular septation, papillary formation, or mural nodule or mass. The normal ovarian stroma, smooth wall or smooth septation, or non-enhancing internal debris is not defined as solid tissue. If solid tissue enhances with a low level type 1 time-intensity curve, the lesion is scored as AdnexMR score 3.

4. If solid tissue is present and it enhances with a timeintensity curve type 2 , the lesion is considered indeterminate on MRI, AdnexMR score 4.

5. If solid tissue is present and it enhances with a time-intensity type 3 or if there is peritoneal disease, then the lesion is highly likely malignant and the AdnexMR score is 5 .

By following this approach, most masses can be allocated a score according to the risk of malignancy, allowing the best clinical management for the patient.

\section{Take-Home Messages}

- Ultrasound is highly effective in the diagnosis of adnexal masses and can direct the treatment approach in most cases.

- It is important for radiologists to recognize the features of common benign and physiological findings.

- MRI is a second-line imaging modality which can be helpful in the characterization of sonographically indeterminate masses, acting as a problemsolving tool.

- For MRI, we recommend an algorithmic approach which includes DWI and contrast-enhanced sequences with DCE as the preferred contrast technique.

\section{References}

1. Levine D, Brown DL, Andreotti RF, et al. Management of asymptomatic ovarian and other adnexal cysts imaged at US: Society of Radiologists in Ultrasound Consensus Conference Statement. Radiology. 2010;256:943-54.

2. Buy JN, Ghossain MA, Hugol D, et al. Characterization of adnexal masses: combination of color Doppler and conventional sonography compared with spectral Doppler analysis alone and conventional sonography alone. AJR Am J Roentgenol. 1996;166: 385-93.

3. Hricak H, Chen M, Coakley FV, et al. Complex adnexal masses: detection and characterization with MRI - multivariate analysis. Radiology. 2000;214:39-46.

4. Timmerman D, Valentin L, Bourne TH, International Ovarian Tumor Analysis (IOTA) Group, et al. Terms, definitions and measurements to describe the sonographic features of adnexal tumors: a consensus opinion from the International Ovarian Tumor Analysis (IOTA) Group. Ultrasound Obstet Gynecol. 2000;16:500-5.

5. Kaijser J, Vandecaveye V, Deroose CM, et al. Imaging techniques for the pre-surgical diagnosis of adnexal tumours. Best Pract Res Clin Obstet Gynaecol. 2014;28:683-95.

6. Timmerman D, Ameye L, Fischerova D, et al. Simple ultrasound rules to distinguish between benign and malignant adnexal masses before surgery: prospective validation by IOTA group. BMJ. 2010;341:c6839.

7. Forstner R, Thomassin-Naggara I, Cunha TM, et al. ESUR recommendations for MR imaging of the sonographically indeterminate adnexal mass: an update. Eur Radiol. 2017;27:2248-57.

8. Grab D, Flock F, Stohr I, et al. Classification of asymptomatic adnexal masses by ultrasound, magnetic resonance imaging, and positron emission tomography. Gynecol Oncol. 2000;77:454-9.

9. Huber S, Medl M, Baumann L, et al. Value of ultrasound and magnetic resonance imaging in the preoperative evaluation of suspected ovarian masses. Anticancer Res. 2002;22:2501-7.

10. Sohaib SA, Mills TD, Sahdev A, et al. The role of magnetic resonance imaging and ultrasound in patients with adnexal masses. Clin Radiol. 2005;60:340-8.

11. Bernardin L, Dilks P, Liyanage S. Effectiveness of semi-quantitative multiphase dynamic contrast-enhanced MRI as a predictor of malignancy in complex adnexal masses: radiological and pathological correlation. Eur Radiol. 2012;22:880-90. 
12. Thomassin-Naggara I, Darai E, Cuenod CA, et al. Dynamic contrast-enhanced magnetic resonance imaging: a useful tool for characterizing ovarian epithelial tumors. J Magn Reson Imaging. 2008;28:111-20.

13. Thomassin-Naggara I, Bazot M, Darai E. Epithelial ovarian tumors: value of dynamic contrast-enhanced MR imaging and correlation with tumor angiogenesis. Radiology. 2008;248:148-59.

14. Thomassin-Naggara I, Daraï E, Cuenod CA, et al. Contribution of diffusion-weighted MR imaging for predicting benignity of complex adnexal masses. Eur Radiol. 2009;19:1544-52.

15. Thomassin-Naggara I, Toussaint I, Perrot N, et al. Characterization of complex adnexal masses: value of adding perfusion- and diffusion-weighted MR imaging to conventional MR imaging. Radiology. 2011;258:793-803.

16. Thomassin-Naggara I, Aubert E, Rockall A, et al. Adnexal masses: development and preliminary validation of an MR imaging scoring system. Radiology. 2013;267:432-43.

17. Forstner R, Sala E, Kinkel K, Spencer JA. ESUR guidelines: ovarian cancer staging and follow-up. Eur Radiol. 2010;20:2773-80.

18. Bhosale RP, Javitt CM, et al. ACR appropriateness criteria ${ }^{\circledR}$ acute pelvic pain in the reproductive age group. Ultrasound $\mathrm{Q}$. 2016;32:108-15.

19. Prabhakar HB, Kraeft JJ, Schorge JO, et al. FDG PET-CT of gynecologic cancers: pearls and pitfalls. Abdom Imaging. 2015;40:2472.

20. Dias JL, Veloso Gomes F, Lucas R, Cunha TM. The shading sign: is it exclusive of endometriomas? Abdom Imaging. 2015;40:2566-72.
21. Corwin MT, Gerscovich EO, Lamba R, et al. Differentiation of ovarian endometriomas from hemorrhagic cysts at MR imaging: utility of the T2 dark spot sign. Radiology. 2014;271:126-32.

22. Yamashita Y, Hatanaka Y, Torashima M, Takahashi M, Miyazaki $\mathrm{K}$, Okamura $\mathrm{H}$. Mature cystic teratomas of the ovary without fat in the cystic cavity: MR features in 12 cases. AJR Am J Roentgenol. 1994;163:613-6.

23. Rha SE, Byun JY, Jung SE, et al. Atypical CT and MRI manifestations of mature ovarian cystic teratomas. AJR Am J Roentgenol. 2004; 183:743-50.

24. Jung SE, Lee JM, Rha SE, Byun JY, Jung JI, Hahn ST. CT and MRI of ovarian tumors with emphasis on the differential diagnosis. Radiographics. 2002;22:1305-25.

25. Lengyel E. Ovarian cancer development and metastasis. Am J Pathol. 2010;177:1053-64.

26. Imaoka I, Wada A, Kaji Y, et al. Developing an MR imaging strategy for diagnosis of ovarian masses. Radiographics. 2006;26:1431-48.

27. Horta M, Cunha TM. Sex cord-stromal tumors of the ovary: a comprehensive review and update for radiologists. Diagn Interv Radiol. 2015;21:277-86.

28. Lalwani N, Prassad SR, Vikram R, Shanboghue AK, et al. Histologic, molecular, and cytogenetic features of ovarian cancers: implication for diagnosis and treatment. Radiographics. 2011;31:625-46.

29. Zhao SH, Quiang JW, Zhang GF, Wang SJ, Qiu HJ, Wang L. MRI in differentiating ovarian borderline from benign mucinous cystadenoma: pathologic correlation. J Magn Reson Imaging. 2014;39:162-6.

Open Access This chapter is licensed under the terms of the Creative Commons Attribution 4.0 International License (http://creativecommons. org/licenses/by/4.0/), which permits use, sharing, adaptation, distribution and reproduction in any medium or format, as long as you give appropriate credit to the original author(s) and the source, provide a link to the Creative Commons license and indicate if changes were made.

The images or other third party material in this book are included in the book's Creative Commons license, unless indicated otherwise in a credit line to the material. If material is not included in the book's Creative Commons license and your intended use is not permitted by statutory regulation or exceeds the permitted use, you will need to obtain permission directly from the copyright holder. 